\title{
FLUID-STRUCTURE INTERACTION OF FOUR RIGIDLY-CONNECTED SQUARE CYLINDERS IN STEADY FLOW
}

\author{
Guoqiang Tang, Mingyin Duan, Lin Lu \\ State Key Laboratory of Coastal and Offshore Engineering \\ Dalian University of Technology, Dalian 116024, China \\ LuLin@dlut.edu.cn \\ MiNG ZHAO \\ School of Computing, Engineering and Mathematics, \\ Western Sydney University, Penrith, NSW 2751, Australia
}

[Received: November 5, 2018; Accepted: March 19, 2019]

\begin{abstract}
Dynamic responses of four rigidly-connected square cylinders in a square configuration subjected to two-dimensional steady flow of a constant property Newtonian fluid were investigated numerically. The focus of the present study is to investigate the effects of the angle of attack $\alpha$ on the dynamic responses by varying $\alpha$ from $0^{\circ}$ to $15^{\circ}$ in intervals of $2.5^{\circ}$ at a fixed $L=4$ ( $L$ is the non-dimensional center-to-center distance between two adjacent square cylinders, normalized by the side length of the square cylinder $B$ ). For each $\alpha$, the reduced velocity $\left(V_{r}\right)$ ranges from 1 to 40 . The Reynolds number $R e$, mass ratio $m^{*}$ and structural damping ratio $\zeta$ maintain constants of 180, 10 and 0 , respectively. Numerical results show that the angle of attack $\alpha$ has a significant influence on the dynamic response. When $\alpha \leq 5^{\circ}$, galloping occurs in addition to vortex-induced vibration (VIV), while it weakens for $\alpha=7.5^{\circ}$ and $10^{\circ}$, and finally disappears as $\alpha=12.5^{\circ}$ and $15^{\circ}$, leaving only VIV response. The effects of $L$ on the responses of the four-square-cylinder oscillating system were also examined for $R e=180, V_{r}=40$, and $\alpha=2.5^{\circ}$. Numerical results show that $L$ affects not only the response displacement but also the vortex shedding mode. Galloping with large response amplitude can happen at either large $L=4$ or small $L=1.5$ and 2 . The response amplitude is relatively small as $2.5 \leq L \leq 3.5$ due to the influence of the flow in the gap between the square cylinders. For the particular case of $L=3.5$, a combined vortex shedding mode is identified, where the vortex shedding from the top row square cylinders behaves as that from an elongated single body while the vortex shedding from the bottom row cylinders presents a co-shedding mode.
\end{abstract}

Mathematical Subject Classification: 34F05, 35R99

Keywords: Vortex-induced vibration, galloping, arbitrary Lagrangian-Eulerian method, Petrov-Galerkin Finite Element Method, square cylinder

\section{INTRODUCTION}

The interaction between multi-body structures and fluid flows has received increasing attention due to its significance in both academic research and practical engineering. It has motivated many investigations to understand flow-induced vibrations. Fluid flow past an elastically-mounted circular cylinder has served as the primary VIV 
problem and has been investigated extensively both numerically and experimentally. Comprehensive reviews on VIV of circular cylinders can be found in 15$]$.

Compared with the VIV of circular cylinders, however, fluid flows past square cylinders have attracted less attention. Previous studies generally focused on fluid flows over fixed square cylinders. The effects of angle of attack on the fluid force, and vortex shedding mode were examined 6 8. As for an elastically-mounted square cylinder placed in a fluid flow, in addition to VIV, galloping is another physical mechanism which occurs when a structure has a non-axisymmetric cross-section 9 11.

A number of experimental investigations have been carried out to understand the physics involved in the dynamic response of a square cylinder. Bokaian and Geoola 12 studied the galloping instability of square cylinders and the corresponding synchronized vortex shedding patterns. The dynamic response of square cylinders was investigated experimentally in a wind tunnel 11]. They reported a galloping response of 1:3 synchronization, where the vortex shedding frequency is three times that of the vibration frequency of the square cylinder. A similar 1:3 synchronization phenomenon was also reported by Wang and Zhou 13, who investigated the effect of attack angle on the dynamic response of a square cylinder. Nemes et al. [14] investigated the effect of angle of attack on the dynamic response experimentally, and found a higher branch of response amplitude at angles of attack of $\alpha \in\left[10^{\circ}, 22.5^{\circ}\right]$. The attack angle of $\alpha=0^{\circ}$ corresponds to the situation in which the flow direction is perpendicular to two side faces of the cylinder. In the higher branch, the maximum amplitude was found to be significantly larger than that in the upper branch of VIV of a circular cylinder, and the vibration frequency synchronized with half of the Strouhal frequency. Zhao et al. 15 conducted a refined experimental study on the dynamic responses of a square cylinder at three different angles of attack, namely $\alpha=0^{\circ}, 20^{\circ}$ and $45^{\circ}$. For $\alpha=0^{\circ}$, a typical galloping response is observed which is characterized by a gradual buildup of the amplitude with the reduced velocity. For $\alpha=45^{\circ}$, the dynamic response is dominated by VIV. Similar to the findings of Nemes et al. 14, Zhao et al. [15] also observed a higher branch at $\alpha=20^{\circ}$, which is confirmed to be a sub-harmonic mode. In the higher branch, the spatial-temporal symmetry of the vortex shedding is broken, leading to a non-zero mean lift force and the high-amplitude branch.

A number of numerical studies have also been conducted to investigate the dynamic response of a square cylinder. Sen and Mittal [16 conducted numerical simulations of fluid flow past a square cylinder at $\alpha=0^{\circ}$. They found that the response amplitude with the reduced velocity can be divided into the primary lock-in, desynchronization and galloping regimes. It was observed that the vortex shedding mode is the $2 S$ mode in the primary lock-in regime, and $2 \mathrm{~S}$ or $2 \mathrm{P}$ modes in the galloping regime. The $2 \mathrm{~S}$ mode means that two single vortices are shed from the square cylinder during one vibration period, while the $2 \mathrm{P}$ mode means that two pairs of vortices are formed and shed every vibration cycle, according to the definition of Williamson and Roshko [17. Later on, Sen and Mittal [18] examined the effects of the mass ratio on the dynamic response of a square cylinder at low Reynolds numbers. The mass ratios of $m^{*}=1$, 
5, 10 and 20 were considered in the numerical simulations. The results indicate that only VIV response appears as $m^{*}=1$. For $m^{*} \geq 5$, galloping branch is observed in addition to VIV. Joly et al. 19] numerically studied the influence of $\alpha$ and $m^{*}$ on the dynamic response of a square cylinder, and developed a sinusoidal quasi-steady model to predict the galloping response. Zhao et al. [20] examined the effects of $\alpha$ on the VIV response of a square cylinder with $\alpha=0^{\circ}, 22.5^{\circ}$ and $45^{\circ}$. The numerical results suggested that the angle of attack affects not only the response amplitudes but also the vortex shedding modes. The dynamic responses of square and rectangular cylinders were numerically studied by Zhao 21] and Cui et al. 22. Different response regimes and vortex shedding modes were reported according to the aspect ratio, which is defined as the cylinder dimension in the cross-flow direction to that in the inline direction.

Compared with the studies of the dynamic responses of an elastically-mounted square cylinder, investigations on fluid multiple square cylinders are fewer and less documented in the literature. The very common configuration of multiple square cylinders is the square arrangement of four square cylinders such as the legs of the tension leg platform in offshore oil and gas engineering. Liu et al. 23. carried out laboratory tests to study the fluid dynamic characteristics around four stationary square-cross-section cylinders in a square arrangement at high Reynolds numbers. The effects of the angle of attack $\alpha$ and the center-to-center distance $L$ between the cylinders on the fluid forces as well as the vortex shedding frequency were investigated. So far, inadequate attention has been paid to the dynamic response of four rigidly-connected square cylinders. In the present study, the dynamic responses of the four rigidly-connected square cylinders in two-dimensional steady flow of a constant property Newtonian fluid were investigated numerically. The aim of this study is to investigate the effects of $\alpha$ on the dynamic responses. The two-dimensional incompressible Navier-Stokes (N-S) equations are solved by a Petrov-Galerkin Finite Element Method (PG-FEM) to predict the fluid flow. An arbitrary Lagrangian-Eulerian (ALE) method is employed to deal with the moving boundaries of the square cylinders. The non-dimensional center-to-center distance between two adjacent square cylinders is fixed as $L=4$ initially. Numerical simulations were performed with a constant Reynolds number $R e=180$. The Reynolds number is defined as $R e=U B / v$, where $v$ is the kinematic viscosity of the fluid, $B$ is the side length of the square cylinder and $U$ is the free-stream velocity. The angle of attack $\alpha$ ranges from $0^{\circ}$ to $15^{\circ}$ with an interval of $2.5^{\circ}$, and the reduced velocity $V_{r}$ varies from 1 to 40 with an increment of 1 .

The remainder of this article is organized as follows. The numerical method employed in this study is introduced in Section 2. The necessary mesh dependent tests are presented in Section 3. The numerical results are shown and discussed in Section 4 , and conclusions are drawn in Section 5.

\section{NUMERICAL METHOD}

Figure 1 shows a sketch of the computational domain for simulating the dynamic responses of the four rigidly-connected square cylinders in a square configuration in 
steady flow. A coordinate system is defined with its origin fixed at the center of the four-cylinder system with the x-coordinate pointing to the flow direction. The set of four rigidly-connected square cylinders is allowed to oscillate in both the cross-flow and in-line directions. The natural frequencies in the $x$ - and $y$-directions are the same. The Reynolds number is set as $R e=180$ throughout the numerical simulations, hence the flow is within the laminar regime so that two-dimensional numerical simulation is appropriate to reveal the potential physical mechanism.

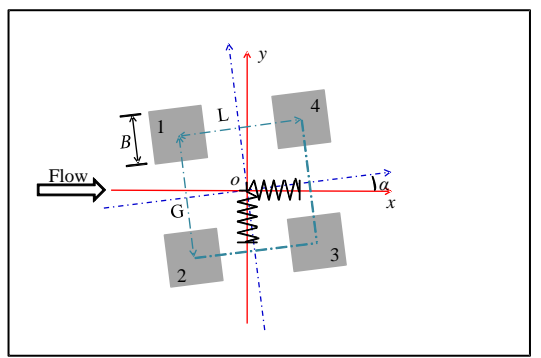

Figure 1. Numerical setup for fluid flow past four elastically supported rigidly connected square cylinders

In this study, the non-dimensional variables of velocity $(u, v)$, the time $t$, the coordinate $(x, y)$ and the pressure $p$ are normalized as $(u, v)=\left(u^{\prime}, v^{\prime}\right) / U, t=t^{\prime} U / B$, $(x, y)=\left(x^{\prime}, y^{\prime}\right) / B$, and $p=p^{\prime} /\left(\rho U^{2}\right)$, respectively, where the prime symbol represents the dimensional variables, $B$ is the side length of the square cylinder, $\rho$ is the fluid density and $U$ is the free-stream velocity. The ALE method is introduced to deal with the moving boundaries induced by the oscillations of the rigidly-connected square cylinders. The non-dimensional governing equations in the ALE scheme are expressed as

$$
\begin{aligned}
\frac{\partial u_{i}}{\partial x_{i}} & =0 \\
\frac{\partial u_{i}}{\partial t}+\left(u_{j}-u_{j, \text { mesh }}\right) \frac{\partial u_{i}}{\partial x_{j}} & =-\frac{\partial p}{\partial x_{i}}+\frac{1}{R e} \frac{\partial^{2} u_{i}}{\partial x_{i} \partial x_{j}} .
\end{aligned}
$$

where $x_{i}$ is the Cartesian coordinate with $x_{1}=x$ and $x_{2}=y, u_{i}$ is the velocity component in the $x_{i}$ direction and $u_{j \text {,mesh }}$ is the velocity of the moving computational mesh. The N-S equations are solved by the PG-FEM, which was same as in Zhao et al. 24. The details of the PG-FEM and the computational procedure are well documented in 24] and will not be repeated in this article.

The motion of the four rigidly connected square cylinders is predicted by solving the following linear oscillator equation

$$
\frac{\partial^{2} X_{i}}{\partial t^{2}}+4 \pi \zeta F_{n} \frac{\partial X_{i}}{\partial t}+\left(2 \pi F_{n}\right)^{2} X_{i}=\frac{C_{i}}{2 m^{*}}
$$

where $X_{i}$ is the non-dimensional displacement of the square cylinders, normalized by the side length of the square cylinder, in the $x_{i}$-direction. $m^{*}=m / m_{s}$ is the mass ratio with $m$ being the total mass of the square cylinders and $m_{s}$ representing the displaced mass of the fluid. In the present study, the mass ratio is set as $m^{*}=10$, 
and $\zeta=c /(4 k m)^{1 / 2}$ is the damping ratio, where $c$ and $k$ are the structural damping coefficient and the spring stiffness, respectively. To investigate possible large amplitude response, $\zeta=0$ is adopted in the numerical simulations. In equation (3), $F_{n}=f_{n} B / U$ is the dimensionless structural natural frequency with $f_{n}$ being the structural natural frequency, $C_{i}$ is the total hydrodynamic coefficient on the square cylinders in the $x_{i}$-direction, which is defined as $C_{i}=2 F_{i} /\left(\rho B U^{2}\right)$ with $F_{i}$ being the total hydrodynamic force in the $x_{i}$-direction. In the following discussions, the hydrodynamic coefficients in the in-line and cross-flow directions $C_{1}$ and $C_{2}$ are replaced by the drag and lift coefficients $C_{D}$ and $C_{L}$, respectively. In equation (3), $X_{1}$ and $X_{2}$ indicate the displacements in $x$ and $y$ directions, namely $X$ and $Y$, respectively, and they are given as

$$
\begin{aligned}
& \frac{\partial^{2} X}{\partial t^{2}}+4 \pi \zeta F_{n} \frac{\partial X}{\partial t}+\left(2 \pi F_{n}\right)^{2} X=\frac{C_{D}}{2 m^{*}}, \\
& \frac{\partial^{2} Y}{\partial t^{2}}+4 \pi \zeta F_{n} \frac{\partial Y}{\partial t}+\left(2 \pi F_{n}\right)^{2} Y=\frac{C_{L}}{2 m^{*}} .
\end{aligned}
$$

Under the ALE description, the nodal coordinates inside the computational domain should be moved according to the motion of the square cylinders. After each computational time step, the mesh is updated by solving the following governing equation 25 27:

$$
\Delta \cdot\left(\gamma S_{i}\right)=0
$$

where $S_{i}$ denotes the displacement of the mesh modal point in the $x_{i}$-direction. To avoid severe mesh deformation around the square cylinders, a parameter $\gamma=1 / A$ is introduced to control mesh deformation, where $A$ is the area of the computational mesh 25 27]. The displacements of the computational nodes on the cylinder surface are the same as that of the oscillating square cylinders, while the displacements of the mesh maintain zero for the rest of the boundaries. By assigning the displacements for all the boundaries, equation (6) is solved by a Galerkin FEM.

A rectangular computational domain with a height of $40 B$ in cross-flow direction and a length of $60 B$ in flow direction is adopted, as shown in Figure 1. The values of upstream and downstream lengths are $20 B$ and $40 B$, respectively. The computational domain is discretized into four-node quadrilateral bi-linear elements. The total number of FEM nodes and elements are 55057 and 54400, respectively. The boundary of each square cylinder is divided into 160 uniformly distributed nodes, resulting in a minimum mesh size along the square cylinder of 0.025 . The radial length of the elements next to the square cylinder surface is 0.01 .

The boundary conditions are specified as follows. On the inlet boundary, the horizontal velocity is set to be $u=1$ and the vertical velocity component is zero. No-slip boundary condition is specified on the surfaces of the square cylinders, i.e. the flow velocity is the same as the vibration velocity of the square cylinders. At the outlet, the pressure is set to be zero, and the gradient of the velocity in the flow direction is zero. On the two lateral boundaries, the gradient of the pressure and the velocity in the transverse direction of the flow are zero. The numerical simulations start from an initial condition that the pressure and the velocity are zero in the whole fluid domain. 


\section{Mesh Dependence Study}

The PG-FEM numerical model and computational code developed by Zhao et al. 24] has been used to simulate the dynamic responses of four square cylinders. It has been demonstrated that this numerical model is capable of simulating the VIV of a square cylinder [20], the galloping response of rectangular cylinders 21, 22], the wake-induced vibrations of two tandem cylinders [28], and the VIV of four rigidly connected circular cylinders 29,30 . The capability of the numerical model has been validated in $16,31,33$ and will not be repeated here.

A mesh dependent study was performed to demonstrate that the mesh used in this study is fine enough to obtain accurate results. Numerical simulations were performed for a fluid flow over four rigidly connected square cylinders at $R e=180$, $V_{r}=40$ and angles of attack $\alpha=0^{\circ}, 5^{\circ}$ and $10^{\circ}$, covering both the VIV and the galloping responses. The corresponding numerical results were compared based on the two different mesh densities, defined as normal mesh and dense mesh, respectively. The total numbers of the nodes and elements are 55057 and 54400 for the normal mesh, while they are 66777 and 66000 for the dense mesh. Each side of the square cylinders is divided into 40 nodes in the normal mesh and 50 nodes for the dense mesh. Table 1 shows the comparisons of the root mean square displacements $X_{r m s}$ and $Y_{r m s}$, the mean total drag coefficients $C_{D, \text { mean }}$, and the root mean square total drag and lift coefficients of $C_{D, r m s}$ and $C_{L, r m s}$. The total drag and lift coefficients are the sum of the drag and lift coefficients of the four square cylinders, respectively. The results in Table 1 demonstrate that the differences between the numerical results in terms of the two meshes are very small. The maximum difference is less than $4.4 \%$. Hence, the normal mesh was used for the numerical simulations in this study. In addition, numerical simulations of VIV of four separately mounted circular cylinders in an in-line square configuration were conducted in our previous study [30], adopting the same numerical model as used in this study. The sizes of the computational domain, upstream, downstream distances and the time step in the present study are similar to those used in 30 . for the case of the center-to-center distance $L / D=4$, where $D$ is the diameter of the circular cylinder. At the same time, the total numbers of the nodes and elements in the present study are larger than those used in [30]. Hence, it is believed that the numerical results in the present study will not be sensitive to the computational mesh.

Table 1. Root mean square displacements and hydrodynamic forces based on normal mesh and dense mesh

\begin{tabular}{|c|c|c|c|c|c|c|}
\hline Case & Mesh & $X_{r m s}$ & $Y_{r m s}$ & $C_{D, \text { mean }}$ & $C_{D, r m s}$ & $C_{L, r m s}$ \\
\hline \hline$\alpha=0^{\circ}, V_{r}=40$ & Normal mesh & 0.1371 & 0.8581 & 6.4580 & 0.6057 & 1.0084 \\
\hline$\alpha=5^{\circ}, V_{r}=40$ & Normal mesh & 0.2341 & 0.6679 & 5.8013 & 0.5882 & 0.8545 \\
\hline$\alpha=10^{\circ}, V_{r}=40$ & Normal mesh & 0.0367 & 0.0989 & 5.1219 & 0.3543 & 2.0183 \\
\hline$\alpha=0^{\circ}, V_{r}=40$ & Dense mesh & 0.1392 & 0.8501 & 6.4591 & 0.6250 & 1.0261 \\
\hline$\alpha=5^{\circ}, V_{r}=40$ & Dense mesh & 0.2272 & 0.6661 & 5.8355 & 0.5896 & 0.8200 \\
\hline$\alpha=10^{\circ}, V_{r}=40$ & Dense mesh & 0.0383 & 0.0972 & 5.1300 & 0.3555 & 2.0214 \\
\hline
\end{tabular}




\section{Numerical RESUlts AND Discussion}

4.1. Vibration amplitude and frequency for $L=4$. Figure 2 shows the variations of the vibration amplitudes in the $x$ - and $y$-directions with the reduced velocity for $\alpha$ ranging from $0^{\circ}$ to $15^{\circ}$. The vibration amplitudes in the $x$ - and $y$-direction are defined as $A_{x}=\left(X_{\max }-X_{\min }\right) / 2$ and $A_{y}=\left(Y_{\max }-Y_{\min }\right) / 2$, respectively, where the subscripts 'max' and 'min' stand for the maximum and minimum displacements, respectively. Figure 3 shows the variations of the non-dimensional response frequency with the reduced velocity under these angles of attack. In the present study, the non-dimensional response frequency is defined as the ratio of dimensional response frequency of the cross-flow displacement to the structural natural frequency, where the dimensional response frequency is obtained by performing Fast Fourier Transform (FFT) analysis on the time history of the cross-flow displacement. The results shown in Figure 2 demonstrate that for some cases, for example when $\alpha=5^{\circ}$ and $22 \leq V_{r} \leq 25$, the response amplitude has dual values in Figure 2 and so does the frequency in Figure 3 .

For these cases, the vibration amplitudes (empty circles in Figure 2 ) are found to be very small if the simulation is initiated with the zero initial condition with the pressure and fluid velocity being zero. The response amplitude increases with the increasing reduced velocity as $V_{r} \geq 13$ and $\alpha=5^{\circ}$, except $22 \leq V_{r} \leq 25$, where the amplitudes are nearly zero. The increase of the response amplitude with increasing $V_{r}$ is a typical characteristic of galloping. However, it is clear that the response amplitudes and frequencies are out of this trend when $\alpha=5^{\circ}$ and $22 \leq V_{r} \leq 25$. It is likely that the response in the reduced velocity range of $22 \leq V_{r} \leq 25$ depends on the initial condition. To justify this, simulations were carried out for $22 \leq V_{r} \leq 25$ by using the fully developed flows of neighboring reduced velocities as initial conditions where galloping occurs. Specifically, the last step results of $V_{r}=21$ were used as the initial condition for $V_{r}=22$ and 23, and the last step results of $V_{r}=26$ were employed as the initial condition for $V_{r}=24$ and 25. The results using the solution of galloping as the initial conditions are plotted as the solid circles in Figure 2 It is found that the response amplitude and frequency are indeed different from their corresponding values when the zero initial condition is used.

Nemes et al. 14 reported a galloping response of a square cylinder when $\alpha=0^{\circ}$, and VIV response when $\alpha=45^{\circ}$. Sen and Mittal 16 simulated the dynamic response of a square cylinder with $m^{*}=10$ at low Reynolds numbers, and found a primary lock-in regime and a secondary lock-in regime. The response in the primary lock-in regime is very similar to that of a circular cylinder. In the secondary lock-in regime, the response amplitude increases continuously with the increase of the reduced velocity. The vibration in the secondary lock-in regime is essentially galloping. Zhao [21] also reported one VIV regime and one galloping regime for the dynamic responses of square and rectangular cylinders. The VIV regime includes an initial branch and a lower branch. In this study, VIV regime is observed to occur at about $6 \leq V_{r} \leq 9$ for all the simulated angles of attack. However, it is interesting to find that two VIV lock-in regimes are identified when the angle of attack is large enough. For example 

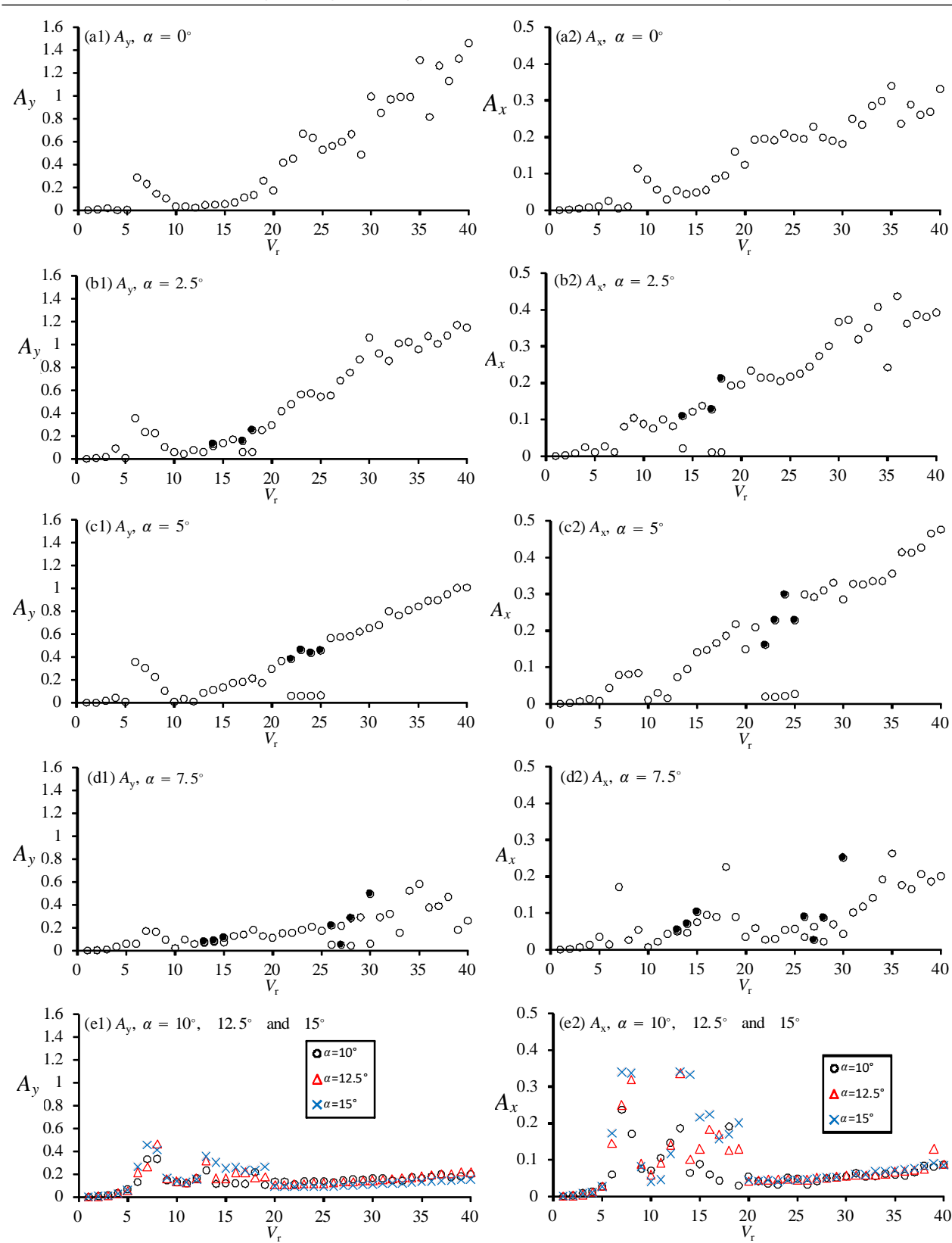

Figure 2. Variations of the vibration amplitudes in the in-line and cross-flow directions with the reduced velocity for different angle of attack values. Empty symbols, still fluid is the initial condition; filled symbols, galloping solution is used as the initial condition 

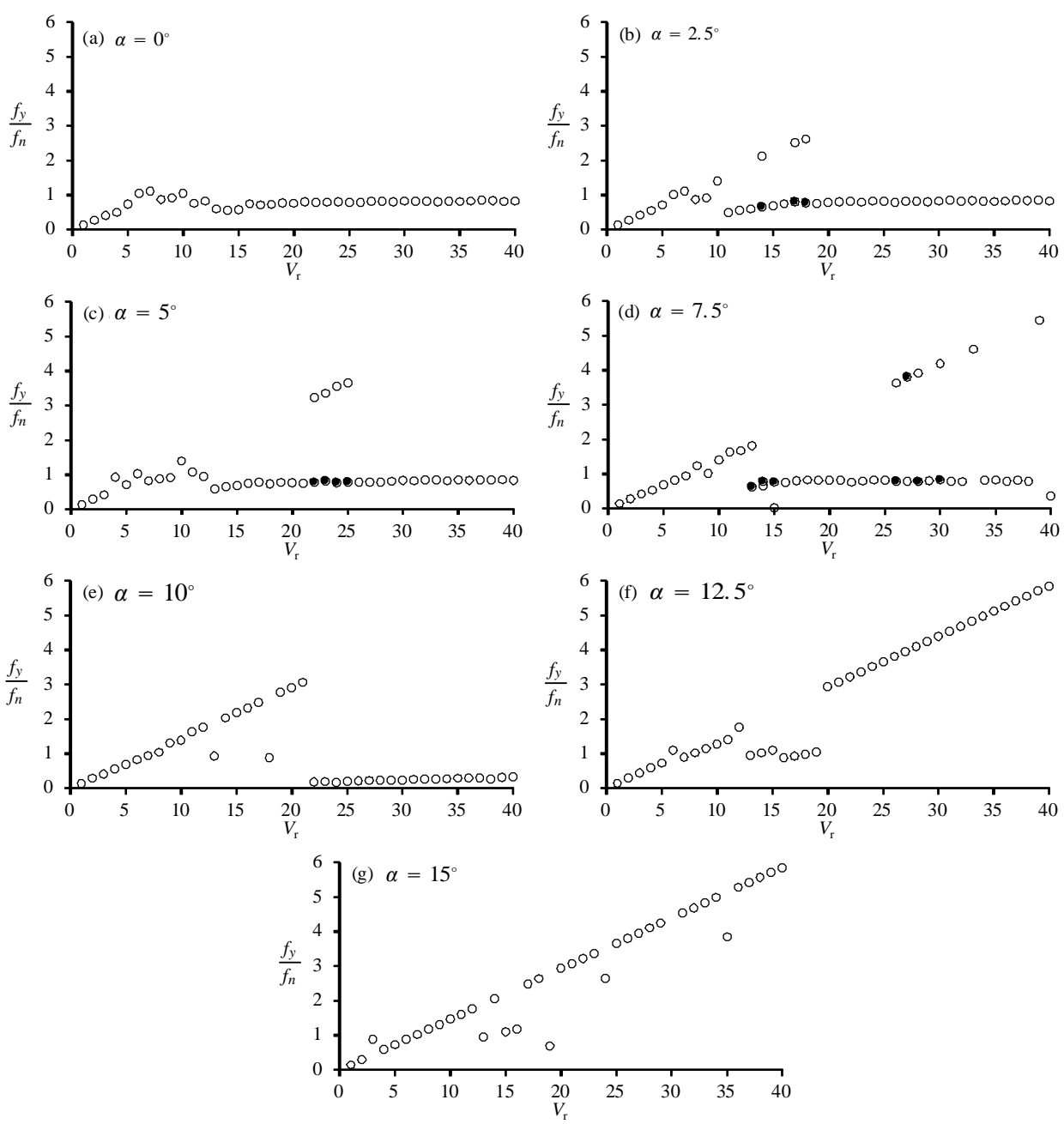

Figure 3. Variation of the vibration amplitudes in the in-line and cross-flow directions with the reduced velocity for different angle of attack values. Empty circles, still fluid is the initial condition; filled circles, galloping solution is used as the initial condition

at $\alpha=12.5^{\circ}$, it is observed that the non-dimensional response frequency is close to but slightly smaller than 1 for $V_{r}=4$ as shown in Figure $3(\mathrm{f})$. It increases gradually to a value slightly greater than 1 as the reduced velocity increases to $V_{r}=9$. This trend is broken at $10 \leq V_{r} \leq 12$. However, as the reduced velocity increases to $V_{r}=13$, lock-in occurs again in the reduced velocity range of $13 \leq V_{r} \leq 19$. As reduced velocity exceeds $V_{r}=20$, the response frequency increases linearly with the increasing reduced velocity. The galloping regimes are clearly observed at $\alpha=0^{\circ}$, 

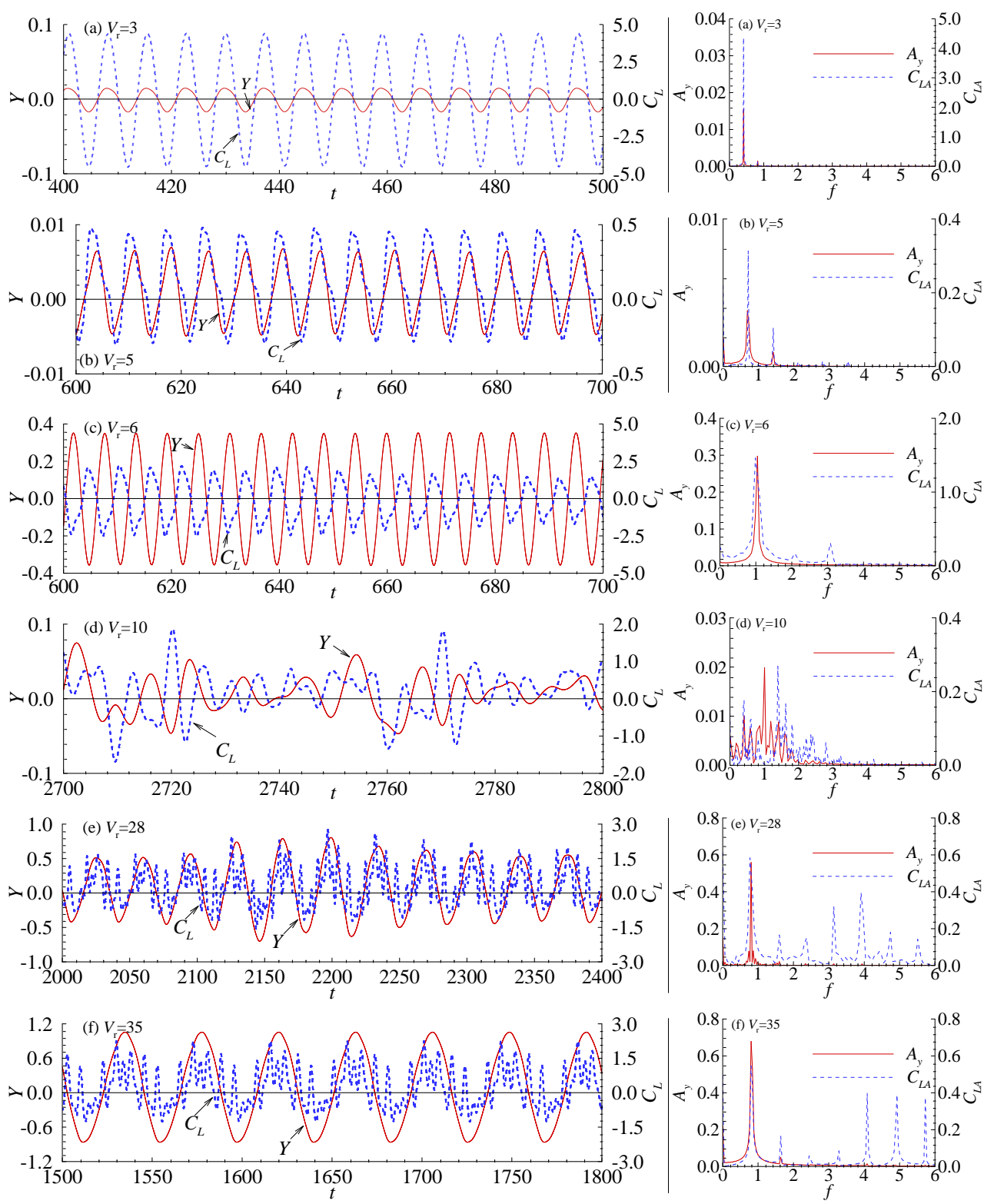

Figure 4. Time histories of cross-flow displacement and lift coefficients (left column) for $\alpha=2.5^{\circ}$ and FFT spectra (right column) for different reduced velocity values

$\alpha=2.5^{\circ}$ and $\alpha=5^{\circ}$ which are characterized by the response frequencies that are slightly lower than 1 at high reduced velocities as shown in Figures 3(a)-(c); such a trend is not clear at $\alpha=7.5^{\circ}$ and disappears at $\alpha=10^{\circ}$. If galloping occurs, the 
vibration amplitudes at high reduced velocities are much larger than the maximum vibration amplitudes in the VIV regime. As $\alpha=0^{\circ}, \alpha=2.5^{\circ}$ and $\alpha=5^{\circ}$, the response amplitude increases with the increase of reduced velocity at $V_{r} \geq 15$, which are in the galloping regime. However, it can be seen that the occurrence of galloping depends on the initial condition for some reduced velocities for $\alpha=2.5^{\circ}$ and $\alpha=5^{\circ}$. As $\alpha \geq 10^{\circ}$, the response amplitude remains consistently small as the reduced velocity exceeds the upper boundary of the VIV lock-in regime.

The results shown in Figure 3 demonstrate that the response frequency of the cylinder in the cross-flow direction is close to the natural frequency in both the VIV and the galloping regimes. However, the correlation between the displacement of the cylinder and the lift coefficient in the VIV regime is different from that in the galloping regime. The time histories and Fast Fourier Transform (FFT) spectra of the vibration displacement and the lift coefficient for $\alpha=2.5^{\circ}$ are shown in Figure 4 as an example to identify the difference between VIV and galloping, where results for $A_{y}$ are shown in red solid lines and those for $C_{L}$ are shown in blue dashed lines. The non-dimensional frequency $f$ is defined as $f=f^{*} B / U$ with $f^{*}$ being the dimensional frequency. The frequencies of the vibration and the lift coefficient of the cylinder are the same in the VIV regime (Figures 4(a), (b) and (c)). In the galloping regime (Figures 4(e) and (f)), a strong high frequency component is observed in the lift fluctuations. However, it does not contribute to the vibration because the vibration amplitude corresponding to this peak frequency is extremely small. By observing the vortex shedding, it was found that the high frequency component in the lift coefficient is actually the vortex shedding frequency. It does not excite VIV because the reduced velocity is outside the lock-in regime for VIV. At $V_{r}=10$ (Figure 4(d)), the vibration changes from the non-galloping regime to the galloping regime. The VIV and galloping regimes cannot be separated from each other at high Reynolds numbers, because the galloping starts from a very small reduced velocity, resulting in an overlap between VIV and galloping regimes [14. The VIV and galloping regimes can be distinctly identified in this study because the Reynolds number is small $(R e=180)$. The VIV and galloping regimes can be separated from each other at low Reynolds numbers because the starting reduced velocity of the galloping regime is higher than the upper boundary of the VIV lock-in regime [16, 21].

It is interesting to see in Figure 3(e) that the response frequency is extremely small and does not vary with the reduced velocity when $\alpha=10^{\circ}$ and $V_{r} \geq 22$. To understand why the frequency is very low, the time histories of the displacements in the $x$ - and $y$-directions for $\alpha=10^{\circ}$ and reduced velocities of $V_{r}=9,17,20$ and 35 are shown in Figure 5. The red lines represent the displacements in the in-line direction while the cross-flow displacements are indicated by the black lines. Regular long-period beating can be seen in Figure 5(a), while the vibration is still dominated by the frequency of the vortex shedding. It can be seen in Figure 5 that the vibration becomes asymmetric and the asymmetry becomes stronger with the increasing reduced velocity. When the reduced velocity increases to 35 (Figure 5 (d)), the beating still exists, while the cylinders vibrate at a high frequency in the crossflow direction. Its mean position also changes periodically in time with an amplitude 

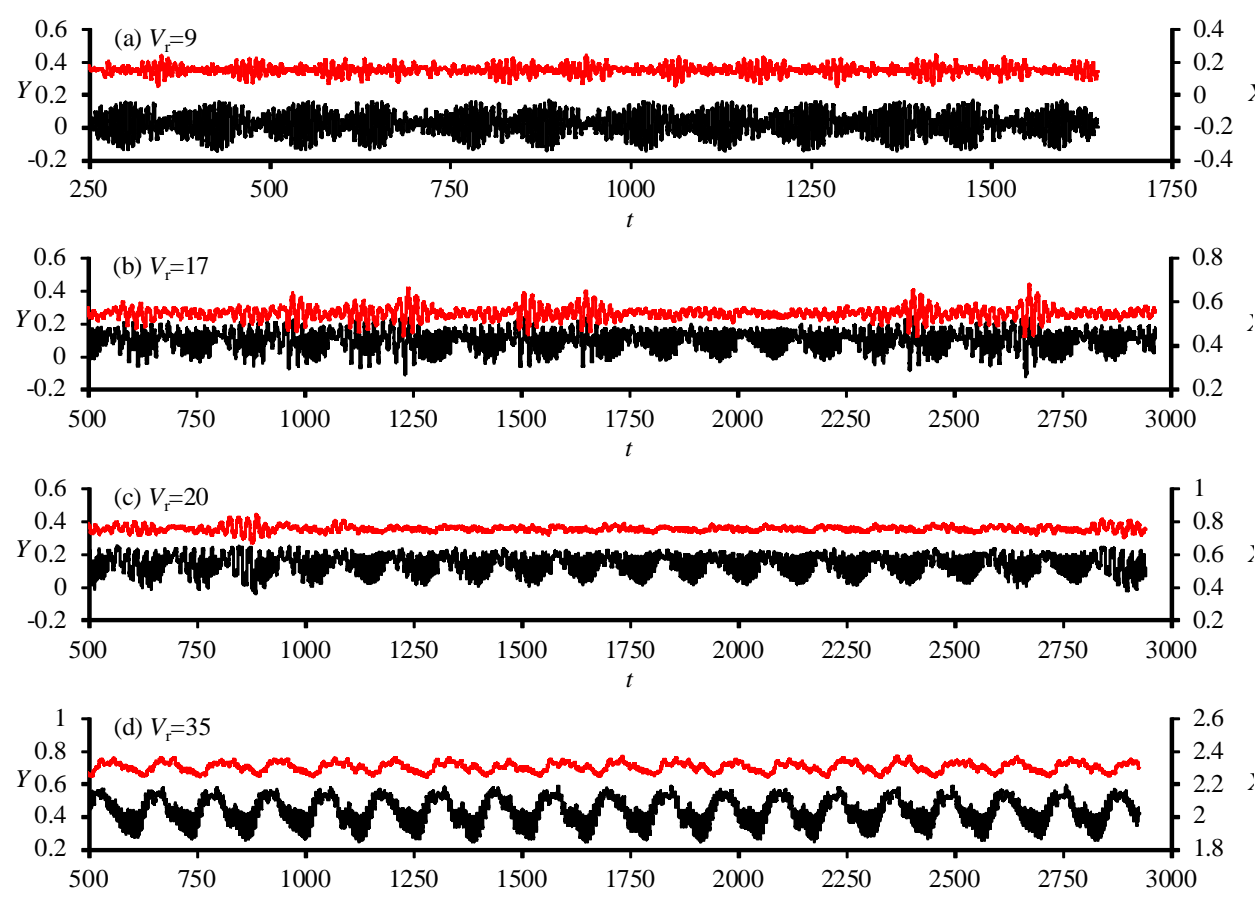

Figure 5. Time histories of the displacements of the cylinder in $x$ - and $y$-directions for $\alpha=10^{\circ}$ for different reduced velocity values. Upper line, displacement in the in-line direction; Lower line, displacement in the cross-flow direction

greater than the high-frequency vibration amplitude. Both the vibration amplitude and frequency for $\alpha=10^{\circ}$ and $V_{r}=35$ are smaller than their counterparts for $\alpha=0^{\circ}$ at the same reduced velocity. The vibration for $V_{r}=9$ in Figure 5(a) is essentially a modulation between a high frequency component and a low frequency component. In Figure 5(d), the vibration is a combination of the modulation of two frequencies and a galloping. Because the galloping amplitude is much smaller than that at $\alpha=0^{\circ}$ and not significantly greater than the VIV amplitude, the vortex-induced vibration can be clearly identified in the time histories.

Figure 6 shows the FFT spectra of the $Y$-displacement and lift coefficient for $\alpha=$ $10^{\circ}$ and two reduced velocities of $V_{r}=9$ and 35. In Figure 6 -displacement, lift coefficients $C_{L}$ and structural natural frequency $f_{n}$ are shown by the black dashed line, red solid line and blue dot-and-dash line, respectively. The modulation between the two signals with distinct frequencies can be identified in Figure 6. The modulation frequencies $\left(f_{m}\right)$ of the $Y$-displacement and the lift coefficient are found to be the same. When $V_{r}=9$, the modulation frequency $\left(f_{m}\right)$ is much smaller than the vortex shedding frequency $\left(f_{s}\right)$. At $V_{r}=35$, in addition to the modulation, a very small frequency exists, which is the frequency of the galloping response $\left(f_{g}\right)$. The vibration is hence a combination of a modulation and a galloping. When galloping occurs, the modulation frequency is the same as the galloping frequency. 

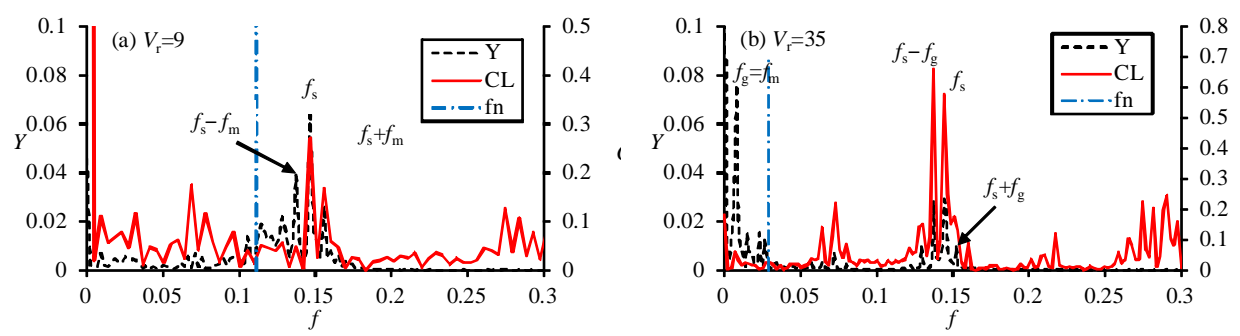

Figure 6. FFT spectra of the $Y$-displacement and lift coefficient for $\alpha=10^{\circ}$ and two typical reduced velocities of $V_{r}=9$ and 35

Based on the above analysis of the vibration amplitude and frequency, we can divide the response into three types according to the angle of attack, namely, galloping $\left(\alpha=0^{\circ}\right.$ to $\left.\alpha=5^{\circ}\right)$, non-galloping or VIV $\left(\alpha \geq 12.5^{\circ}\right)$, and a combined state between them $\left(\alpha=7.5^{\circ}\right.$ and $\left.\alpha=10^{\circ}\right)$. The $Y$-amplitude in the galloping regime increases with the increase of $\alpha$ as shown in Figure 2(a) while the non-galloping vibration, which is outside the VIV lock-in regime, has very small amplitudes. In the combined state, the dynamic responses of the square cylinders are a combination of the weak galloping and the vortex-induced vibration.
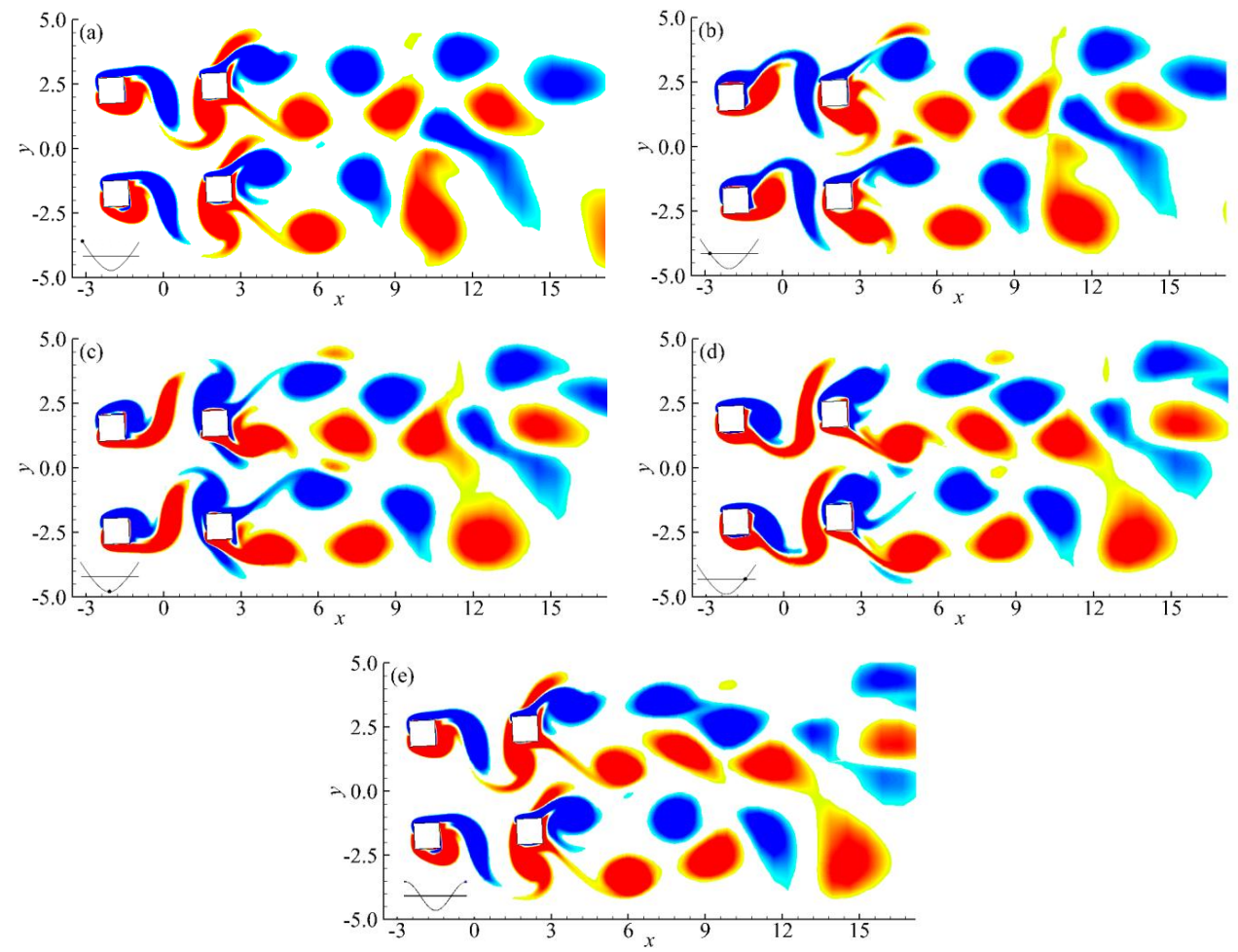

Figure 7. Vorticity contours over one period of oscillation for $\alpha=$ $2.5^{\circ}$ and $V_{r}=6$. (a), $Y=Y_{\max } ;$ (b), $Y=0$, moving down; (c), $Y=Y_{\min } ;(\mathrm{d}), Y=0$, moving up; (e), $Y=Y_{\max }$ 
Figure 7 shows the vorticity contours over one period of oscillation for $\alpha=2.5^{\circ}$ and $V_{r}=6$, which is in the lower branch of the VIV lock-in regime. The instantaneous position of the four-cylinder system on the Y-displacement is shown in each diagram in Figure 7. The first time instant is obtained at $Y=Y_{\max }$ and is followed by $1 / 4$ period of the oscillation of the square cylinders. A pair of vortices is shed from each of the upstream cylinders in one cycle of vibration. The vortices that are shed from the upstream cylinders attack the downstream cylinder and combine with the vortices generated from the downstream cylinders. The combination of the vortices makes the vortex shedding from the two downstream cylinders easy to identify; they are also stronger than the vortices from the upstream cylinders. The number of vortices that are shed from each of the downstream cylinders is a pair. The interaction between the vortices from the top row cylinders and those from the bottom row cylinders appears to be weak. The two rows of vortex street can be clearly seen downstream the cylinders regime.

The instantaneous $Y$-displacement of the four-cylinder system is marked in Figure 8 which shows the vorticity contours over one period of oscillation for $\alpha=2.5^{\circ}$ and $V_{r}=35$ in the galloping regime. The first time instant is obtained at $Y=Y_{\max }$
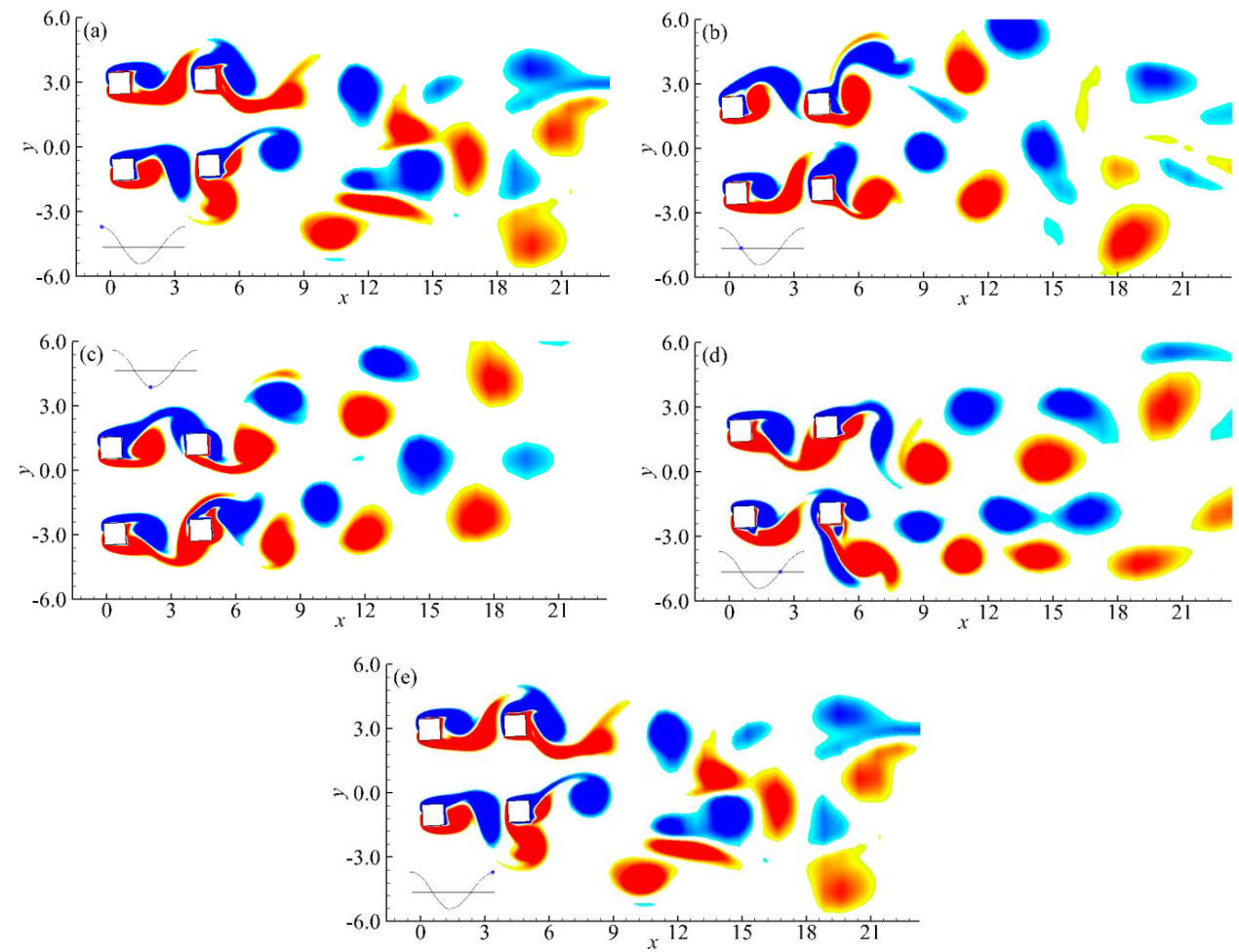

Figure 8. Vorticity contours over one period of oscillation for $\alpha=$ $2.5^{\circ}$ and $V_{r}=35$. (a), $Y=Y_{\max }$; (b), $Y=0$, moving down; (c), $Y=Y_{\min } ;(\mathrm{d}), Y=0$, moving up; (e), $Y=Y_{\max }$ 
(Figure 8(a) and is followed by each $1 / 4$ period of the oscillation of the square cylinders. The number of the vortices that are shed from each of the cylinders in one period of the galloping presents also as a pair. However, there are some differences between the vortices in the VIV lock-in regime (Figure 7) and those in the galloping regime (Figure 8). In the VIV lock-in regime, the shear layers from the two sides of each upstream cylinder form vortices at a further downstream location of the cylinder than those in the galloping regime. Each of the vortices from the upstream cylinders in the VIV lock-in regime is elongated and split into two by the downstream cylinder. The elongation of the vortices delays the vortex shedding and detunes the vortex shedding frequency to the natural frequency. In the galloping regime, the vortices generated from different vortex shedding periods behave differently, because the position of the cylinders at different vortex shedding periods is different. In the galloping regime and while the cylinders moving downwards or upwards, a number of different vortex shedding periods are involved, resulting in a slightly tilted vortex street, as shown in Figures $8(\mathrm{c})$ and (e).

4.2. Effect of center-to-center distance on galloping for $\alpha=2.5^{\circ}$. It has been found that the center-to-center distance $(L)$ has a significant effect on the VIV response of four circular cylinders in an inline square arrangement [30]. This section mainly focuses on the effect of $L$ on the dynamic response. Simulations were performed by varying $L$ from 1.5 to 4 with an increment of 0.5 for a angle of attack with $\alpha=2.5^{\circ}$ and a reduced velocity of $V_{r}=40$. The center-to-center distance $L$ is found to have significant effects on the vibration of the square cylinders. Figure 9 shows the time histories and FFT spectra of the $Y$-displacement and the lift fluctuation for different values of $L$, where the results for the $Y$-displacement are shown in red solid lines and those for lift fluctuation are shown in blue dashed lines.

Figures 10 and 11 show the vorticity contours for $L=1.5$ and 2 , respectively. The position of the cylinder is marked on the $X Y$-trajectory in each diagram of Figures 10 and 11. Based on the time histories and the vortex shedding patterns, it can be seen that the vibrations are typical of the galloping responses for $L=1.5$ and 2 . At $L=1.5$, six vortices (labeled by A-E in Figure 10) are shed from the cylinders in one cycle of vibration. The jet flowing from the gap between the top and bottom rows of the cylinders is very weak and merges into the shear layers from two sides of the cylinder system. The vortex street in the wake of the four square cylinders is very similar to that behind a single structure. As $L=2$, the vibration is a typical galloping response, characterized by a number of vortex shedding periods in one cycle of vibration as shown in Figure 9(b). Because the shear layers generated from the horizontal gap form vortices that are shed from the cylinders, the number of the vortices that are shed from the cylinders in one cycle of vibration for $L=2$ is greater than that for $L=1.5$. The combination of the vortices from the bottom row cylinders with those from the top row cylinders occurs continuously. Each of the vortices A-I in Figure 11 is the combined vortex. For example, the vortices A1 and A2 in Figure 11(a) combine to form a single vortex A, as shown in Figure 11(b). The galloping at $L=1.5$ and 2 results in high amplitude vibrations, as shown in Figure 9 , Due to 
the effects of the jet flow from the horizontal gap between the cylinders, the vibration amplitude at $L=2$ is lower than that at $L=1.5$.
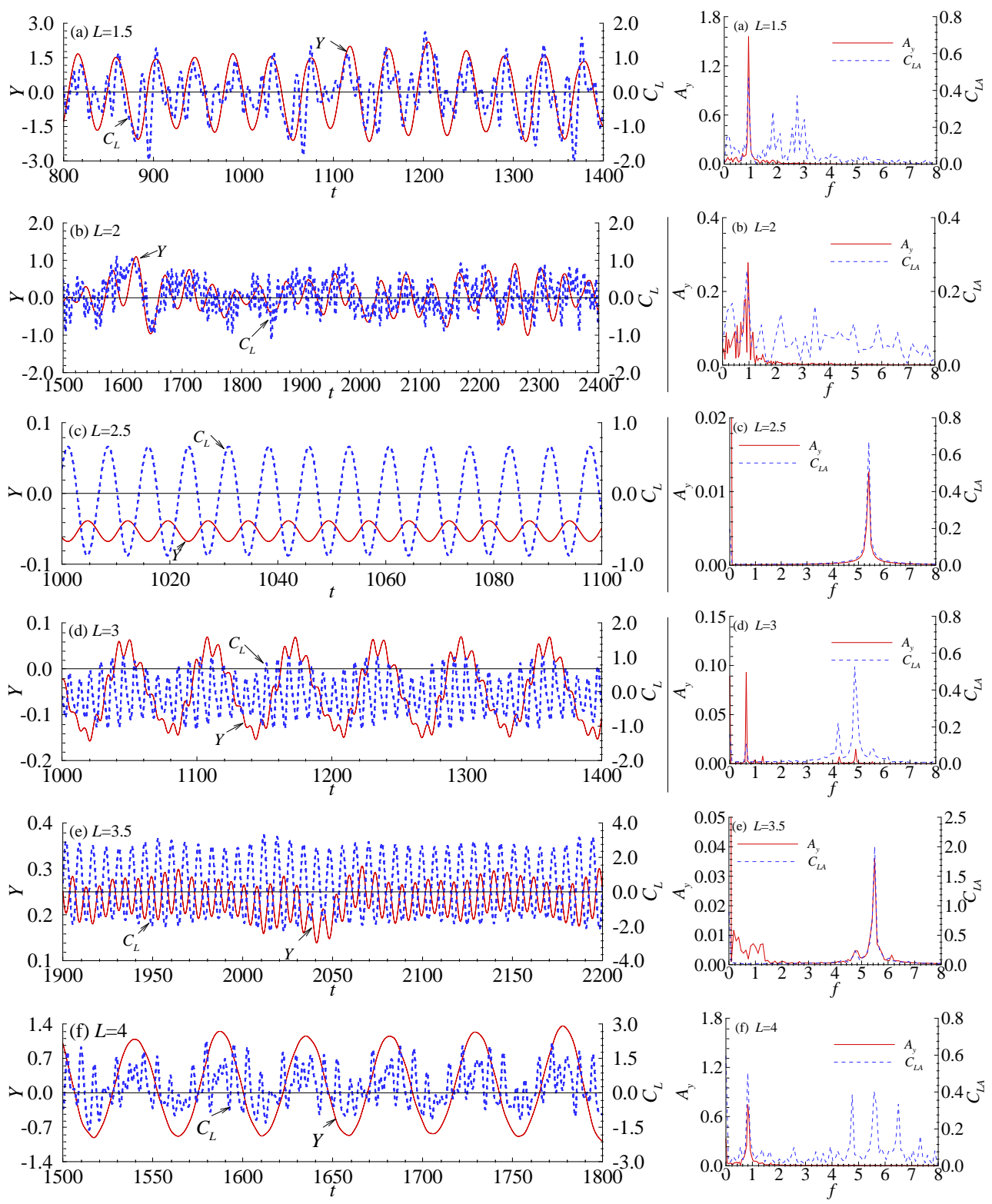

Figure 9. Time histories (left column) and FFT spectra (right column) of the cross-flow displacement and the lift force coefficient for different center-to-center distances $L$ at fixed $\alpha=2.5^{\circ}$ and $V_{r}=40$. Solid line, $Y$-displacement; Dashed line, lift coefficient 

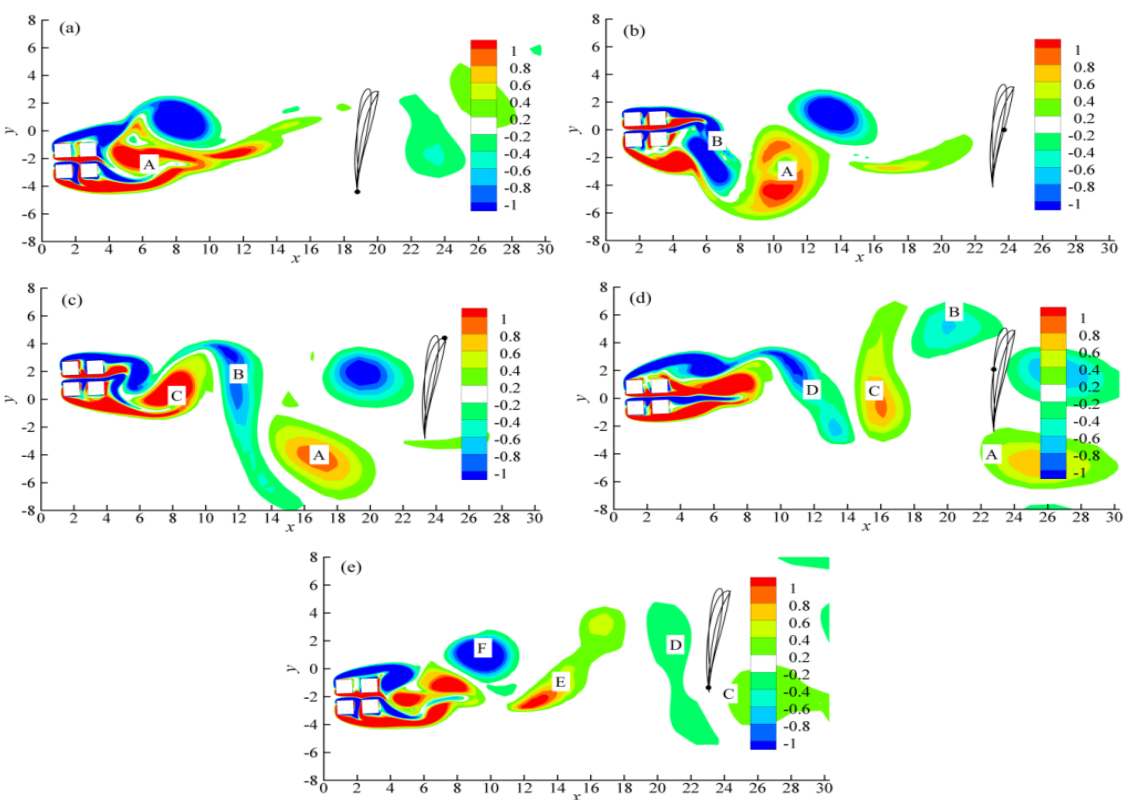

Figure 10. Vorticity contours for $\alpha=2.5^{\circ}, V_{r}=40$ and $L=1.5$. The position of the cylinder is marked on the $X Y$-trajectory in each diagram
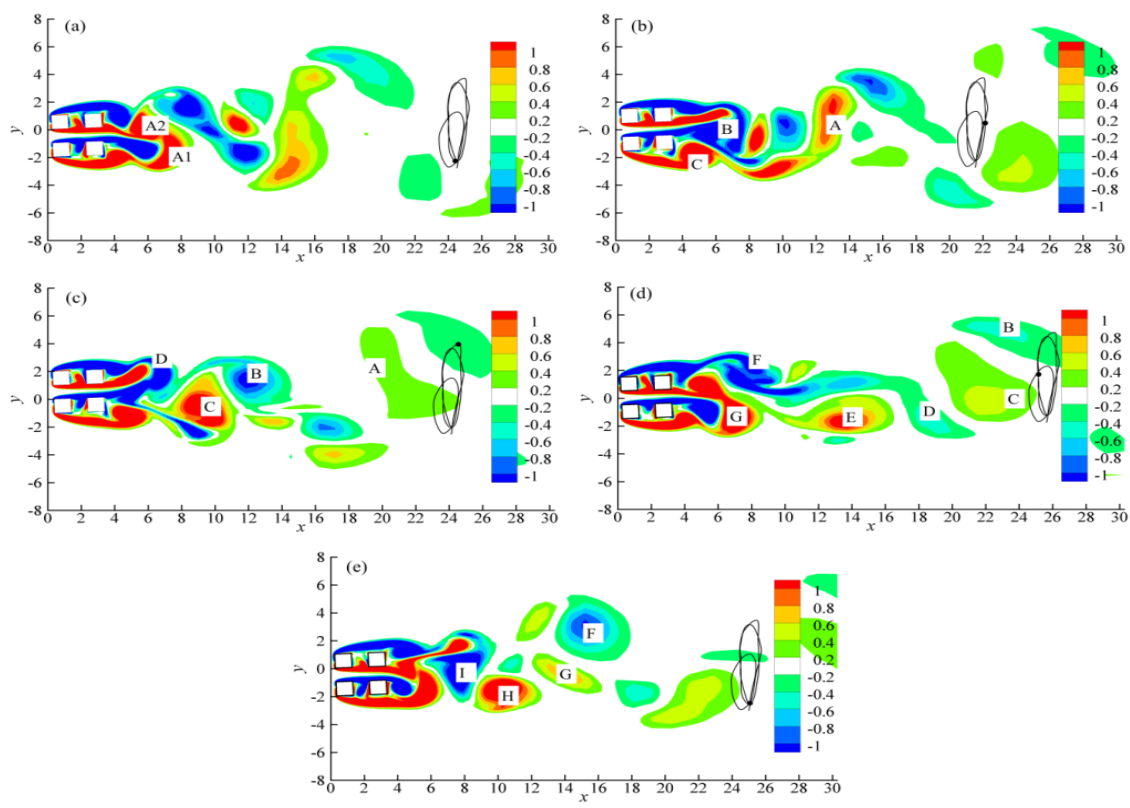

Figure 11. Vorticity contours for $\alpha=2.5^{\circ}, V_{r}=40$ and $L=2$. The position of the cylinder is marked on the $X Y$-trajectory in each diagram. 
Figure 12 shows the vorticity contours for $L=2.5$. The difference between the flows for $L=2$ and 2.5 is that at $L=2.5$. A complete vortex shedding process occurs from each of the downstream cylinders. The combination of the vortices also occurs, which is similar to that for $L=2$. The combination only occurs after the vortices are totally separated from the cylinders. The vibration amplitude is very small and no galloping is observed for $L=2.5$, because the vortex shedding occur from the two upstream cylinders. Each horizontal row of cylinders behaves as a single body that conforms better than a single cylinder. The vortex shedding processes from the two downstream cylinders synchronize perfectly at $L=2.5$, resulting in the very periodic vibration shown in Figure 9 (c).
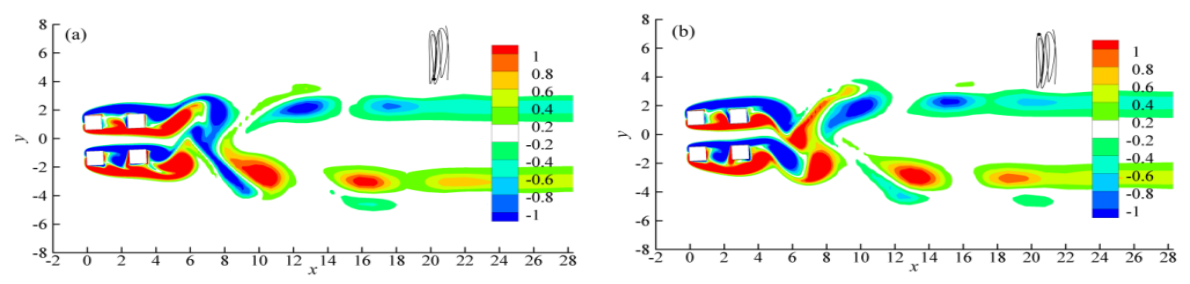

Figure 12. Vorticity contours for $\alpha=2.5^{\circ}, V_{r}=40$ and $L=2.5$. The position of the cylinder is marked on the $X Y$-trajectory in each diagram.

Figures 13 and 14 show the vorticity contours for $L=3$ and 3.5, respectively. When $L$ increases to 3 , the vortices generated from the horizontal gap of the cylinders are strong. This stops the combinations of the vortices. In Figure 13 , the vortices A1 and A2 are shed from the gap almost at the same time and coexist as they are convected downstream. There are a number of vortex shedding periods in one cycle of vibration. When two cylinders are placed in a flow in a tandem arrangement, vortex shedding only occurs from the downstream cylinder, as the two cylinders are very close to each other, leading to a single body vortex shedding flow mode. When the gap between the two cylinders is large enough, vortex shedding occurs from both cylinders and the flow is in a co-shedding mode (Zdravkovich [34]; Zhou and Yiu [35]). It is interesting to see that at $L=3.5$, the vortex shedding from the top row cylinders is in an elongated single body mode, while that from the bottom row cylinders is in a coshedding mode. When four circular cylinders are in an inline square arrangement $\left(\alpha=0^{\circ}\right)$, the combination of single body and co-shedding modes does not occur (Zhao et al. 30]). This is a distinct feature for flow past four cylinders at a small angle of attack. Although the vortices shed from the cylinders are in different modes, the vortex shedding period of the four cylinders synchronizes. This is evidenced by the FFT spectrum shown in Figure 9(e), where the total lift coefficient only has one predominant frequency component. The lift coefficient would have had multiple frequencies if the vortices shedding from the top row cylinders were different from those from the bottom row cylinders. As $L$ is increased to 4 , vortex shedding occurs from all four square cylinders, and the vibration is in a high amplitude galloping mode. The flow patterns for $L=4$ and $V_{r}=40$ are not presented since they are similar to that for $V_{r}=35$ shown in Figure 8 . 

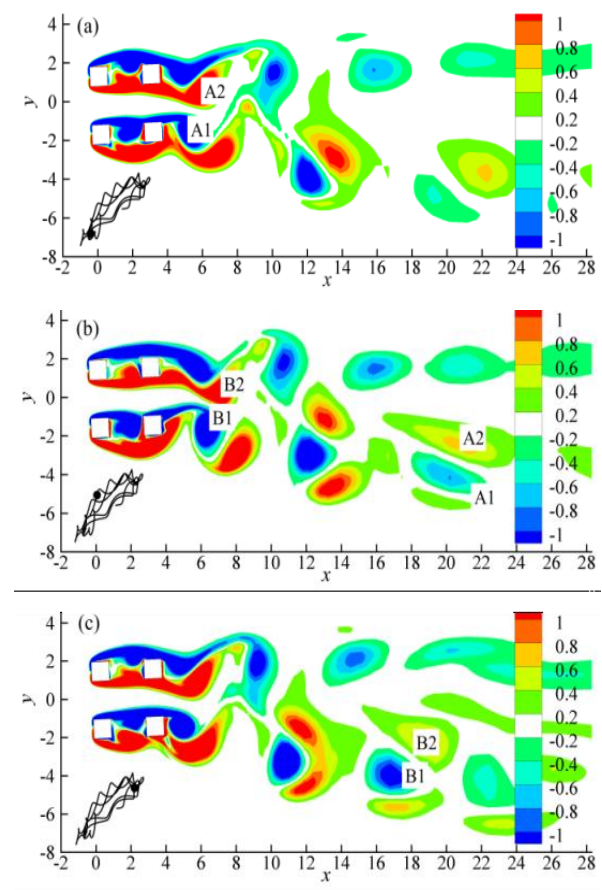

Figure 13. Vorticity contours for $\alpha=2.5^{\circ}, V_{r}=40$ and $L=3$. The position of the cylinder is marked on the $X Y$-trajectory in each diagram.
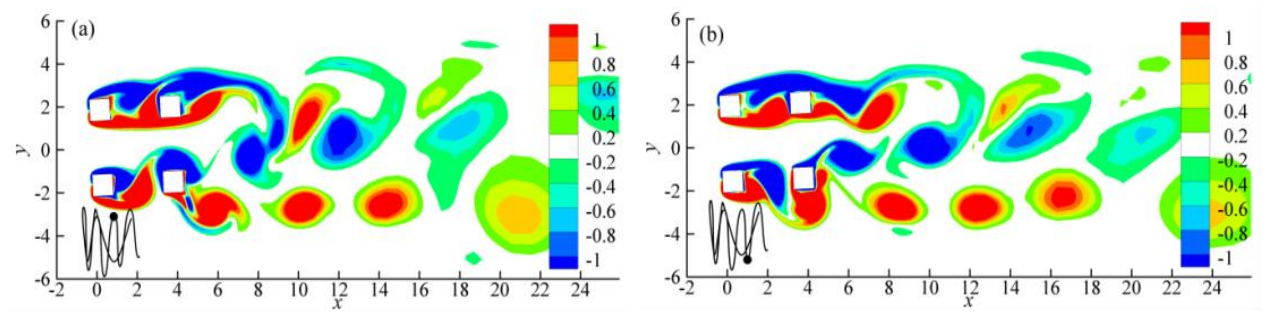

Figure 14. Vorticity contours for $\alpha=2.5^{\circ}, V_{r}=40$ and $L=3.5$. The position of the cylinder is marked on the $X Y$-trajectory in each diagram.

The above numerical results suggest that the distance $L$ has a significant effect on the vibration amplitude and the response frequency through changing the flow mode. If $L$ is either sufficiently large enough $(L=4)$ or sufficiently small ( $L=1.5$ and 2 ), galloping occurs and the vibration amplitude is large. The vibration for very large $L$ comprises galloping responses of four cylinders while the vibration for very small $L$ resembles the response of a single cylinder. The vibration amplitude is reduced significantly for $2.5 \leq L \leq 3.5$ because of the influence from the horizontal gaps of the cylinders. 


\section{Conclusions}

Numerical simulations were conducted to study the flow-induced vibrations of four rigidly-connected cylinders in a square arrangement in two-dimensional steady flow of a constant property Newtonian fluid with low Reynolds number. The conclusions are summarized as follows:

(1) The effect of angle of attack $(\alpha)$

VIV response is observed for all the simulated angles of attack with $L=4$. The galloping occurs when the angle of attack is smaller than $\alpha=5^{\circ}$. VIV and galloping responses can be separated from each other because the galloping happens at critical reduced velocities, which are greater than the upper boundary of the VIV lock-in regime. When the angle of attack is large enough (for example, $\alpha=12.5^{\circ}$ and $15^{\circ}$ ), only VIV responses are observed. As for $\alpha=7.5^{\circ}$ and $10^{\circ}$, the modulation between the vortex shedding frequency and a very low frequency was observed. With the increase of reduced velocity, the low frequency component become more significant, and the dynamic response amplitude increases and finally evolves into galloping.

(2) The effect of center-to-center distance $(L)$

The effects of the center-to-center distance on the dynamic response were examined by simulating the dynamic response at typical $V_{r}=40$ and $\alpha=2.5^{\circ}$. Galloping occurs when $L$ is either large or small. When $L$ is an intermediate value (from 2.5 to 3.5 ), galloping is not observed because the vortex shedding flow is strongly affected by the jet flow from the gap of the cylinders. At $L=3.5$ a combination of the single body vortex shedding mode from the top row cylinders and the co-shedding mode from the bottom row cylinders is observed. This combined vortex shedding mode is believed to be a distinct feature for small angles of attack.

\section{Nomenclature}

angle of attack, non-dimensional center-to-center distance between two adjacent square cylinders, side length of the square cylinder, structural natural frequency, free-stream velocity, reduced velocity, $U / f_{n} B$, kinematic viscosity coefficient, Reynolds number, $U B / v$, total mass of the square cylinders, displaced mass of the fluid, mass ratio, $m / m_{s}$, structural damping coefficient, structural spring stiffness, structural damping ratio, $c /(4 k m)^{1 / 2}$, non-dimensional variables of velocity, 


$\begin{array}{ll}t & \text { time, } \\ p & \text { pressure, } \\ \rho & \text { fluid density, } \\ x_{i} & \text { Cartesian coordinate with } x_{1}=x \text { and } x_{2}=y, \\ u_{i} & \text { velocity component in the } x_{i} \text { direction, } \\ u_{j \text { mesh }} & \text { velocity of the moving computational mesh, } \\ X_{i} & \text { non-dimensional displacement, } \\ F_{n} & \text { dimensionless structural natural frequency, } f_{n} B / U, \\ F_{i} & \text { total hydrodynamic force in the } x_{i} \text {-direction, } \\ C_{i} & \text { total hydrodynamic coefficient on the square cylinders in the } x_{i} \text { - } \\ & \text { direction, } 2 F_{i} /\left(\rho B U^{2}\right), \\ C_{D} & \text { drag coefficient, } \\ C_{L} & \text { lift coefficient, } \\ S_{i} & \text { displacement of the mesh modal point in the } x_{i} \text {-direction, } \\ A & \text { area of the computational mesh, } \\ \gamma & \text { introduced to control mesh deformation, } 1 / A, \\ X_{r m s}, Y_{r m s} & \text { root mean square displacement, } \\ C_{D, m e a n} & \text { mean total drag coefficient, } \\ C_{D, r m s}, C_{L, r m s} & \text { root mean square total drag and lift coefficients, } \\ A_{x} & \text { vibration amplitudes in the } x \text {-direction, }\left(X_{\max }-X_{\min }\right) / 2, \\ A_{y} & \text { vibration amplitudes in the } y \text {-direction, }\left(Y_{\max }-Y_{\min }\right) / 2, \\ X_{\max }, X_{\min }, Y_{\max }, Y_{\min } & \text { maximum and minimum displacements, } \\ X & \text { displacements of the cylinder in } x \text {-direction, } \\ Y & \text { displacements of the cylinder in } y \text {-direction, } \\ f_{m} & \text { modulation frequency, } \\ f_{s} & \text { vortex shedding frequency, } \\ f_{g} & \text { frequency of the galloping response }, \\ f^{*} & \text { dimensional frequency, } \\ f & \text { non-dimensional frequency, } f=f^{*} B / U . \\ & \end{array}$

Acknowledgement. The authors would like to acknowledge supports from the National Key R\&D Program of China (Project ID: 2017YFC1404202) and the National Natural Science Foundation of China (Grant No. 51979031, 51779040 and 51490673).

\section{REFERENCES}

1. P. W. Bearman. "Vortex shedding from oscillating bluff bodies." Annual Review of Fluid Mechanics, 16, (1984), pp. 195-222. DOI: 10.1146/annurev.fl.16. 010184.001211.

2. T. Sarpkaya. "A critical review of the intrinsic nature of vortex-induced vibration." Journal of Fluids and Structures, 19(4), (2004), pp. 389-447. DOI: 10.1016/j·jfluidstructs.2004.02.005

3. C. H. K. Williamson and R. Govardhan. "Vortex-induced vibrations." Annual Review of Fluid Mechanics, 36, (2004), pp. 413-455. DOI: 10.1146/annurev. fluid.36.050802.122128 
4. R. D. Gabbai and H. Benaroya. "An overview of modeling and experiments of vortex-induced vibration of circular cylinders." Journal of Sound and Vbration, 282(3-5), (2005), pp. 575-616. DOI: 10.1016/j.jsv.2004.04.017.

5. C. H. K. Williamson and R. Govardhan. "A brief review of recent results in vortex-induced vibrations." Journal of Wind Engineering and Industrial Aerodynamics, 96(6-7), (2008), pp. 713-735. DOI: 10.1016/j.jweia.2007.06.019.

6. S. C. Luo, X. H. Tong, and B. C. Khoo. "Transition phenomena in the wake of a square cylinder." Journal of Fluids and Structures, 23(2), (2007), pp. 227-248. DOI: $10.1016 / \mathrm{j} \cdot \mathrm{jfluidstructs.2006.08.012.}$

7. G. J. Sheard, M. J. Fitzgerald, and K. Ryan. "Cylinders with square crosssection: wake instabilities with incident angle variation." Journal of Fluid Mechanics, 630(2), (2007), pp. 43-69. DOI: 10.1017/S0022112009006879

8. D. H. Yoon, K. S. Kang, and C. B. Choi. "Flow past a square cylinder with an angle of incidence." Physics of Fluids, 22(4), 043603, (2010). DOI: 10.1063/1. 3388857 .

9. G. V. Parkinson and N. P. H. Brooks. "On the aeroelastic instability of bluff cylinders." Journal of Applied Mechanics-Transactions of the ASME, 28(2), (1961), pp. 252-258. DOI: 10.1115/1.3641663.

10. G. V. Parkinson and J. D. Smith. "The square prism as an aeroelastic non-linear oscillator." Quarterly Journal of Mechanics and Applied Mathematics, 17(2), (1964), 225-239. DOI: 10.1093/qjmam/17.2.125.

11. P. W. Bearman, I. S. Gartshore, D. J. Maull, and G. V. Parkinson. "Experiments on flow-induced vibration of a square-section cylinder." Journal of Fluids and Structures, 1(1), (1987), pp. 19-34. DOI: 10.1016/S0889-9746(87)90158-7.

12. A. R. Bokaian and F. Geoola. "Hydroelastic instabilities of square cylinders." Journal of Sound and Vibration, 92(1), (1984), pp. 117-141. DOI: 10.1016/ 0022-460X (84) 90378-X.

13. Z. J. Wang and Y. Zhou. "Vortex-induced vibration characteristics of an elastic square cylinder on fixed supports." Journal of Fluids Engineering, 127(2), (2004), pp. 241-249. DOI: 10.1115/1.1881693.

14. A. Nemes, J. S. Zhao, D. L. Jacono, and J. Sheridan. "The interaction between flow-induced vibration mechanisms of a square cylinder with varying angles of attack." Journal of Fluids Engineering, 710(11), (2004), pp. 102-130. DOI: 1017/ jfm.2012.353.

15. J. S. Zhao, J. S. Leontini, D. L. Jacono, and J. Sheridan. "Fluid-structure interaction of a square cylinder at different angles of attack." Journal of Fluid Mechanics, 747 (2014), pp. 688-721. DOI: 10.1017/jfm.2014.167.

16. S. Sen and S. Mittal. "Free vibration of a square cylinder at low Reynolds numbers." Journal of Fluids and Structures, 27(5-6), (2011), pp. 875-884. DOI: 10.1016/j.jfluidstructs.2011.03.006.

17. C. H. K. Williamson and A. Roshko. "Vortex formation in the wake of an oscillating cylinder." Journal of Fluids and Structures, 2(4), (1988), pp. 355-381. DOI: $10.1016 / \mathrm{S} 0889-9746$ (88) 90058-8. 
18. S. Sen and S. Mittal. "Vortex formation in the wake of an oscillating cylinder." Journal of Fluids and Structures, 54(4), (2015), pp. 661-678. DOI: $10.1016 / \mathrm{j}$. jfluidstructs.2015.01.006.

19. A. Joly, S. Etienne, and D. Pelletier. "Galloping of square cylinders in crossflow at low Reynolds numbers." Journal of Fluids and Structures, 28(1), (2012), pp. 232-243. DOI: $10.1016 / \mathrm{j} \cdot \mathrm{jfluidstructs.2011.12.004.}$

20. M. Zhao, L. Cheng, and T. M. Tong. "Numerical simulation of vortex-induced vibration of a square cylinder at a low Reynolds number." Physics of Fluids, 25(2), 023603 (2013). DOI: 10.1063/1.4792351.

21. M. Zhao. "Flow-induced vibrations of square and rectangular cylinders at low Reynolds number." Fluid Dynamics Research, 47(2), (2015). DOI: $10.1088 /$ 0169-5983/47/2/025502.

22. Z. D. Cui, M. Zhao, B. Teng, and L. Cheng. "Two-dimensional numerical study of vortex-induced vibration and galloping of square and rectangular cylinders in steady flow." Ocean Engineering, 106(9) (2015), pp. 189-206. DOI: 10.1016/j . oceaneng.2015.07.004

23. M. Y. Liu, L. F. Xiao, and L. J. Yang. "Experimental investigation of flow characteristics around four square-cylinder arrays at subcritical Reynolds numbers." International Journal of Naval Architecture and Ocean Engineering, 7(5) (2015), pp. 906-919. DOI: 10.1515/ijnaoe-2015-0063.

24. M. Zhao, L. Cheng, B. Teng, and G. H. Dong. "Hydrodynamic forces on dual cylinders of different diameters in steady currents." Journal of Fluids and Structures, 23(1) (2007), pp. 59-83. DOI: 10.1016/j · jfluidstructs.2006.07.003.

25. M. Zhao and L. Cheng. "Numerical simulation of two-degree-of-freedom vortexinduced vibration of a circular cylinder close to a plane boundary." Journal of Fluids and Structures, 27(7) (2011), pp. 1097-1110. DOI: 10.1016/j·jfluidstructs . 2011.07.001.

26. M. Zhao, L. Cheng, and L. Lu. "Vortex induced vibrations of a rotating circular cylinder at low Reynolds number." Physics of Fluids, 26, 073602, (2014). DOI: $10.1063 / 1.4886196$

27. M. Liu, L. Lu, B. Teng, M. Zhao, and G. Q. Tang. "Numerical modeling of local scour and forces for submarine pipeline under surface waves." Coastal Engineering, 116(10) (2016), pp. 275-288. DOI: 10.1016/j.coastaleng.2016.05.003.

28. M. Zhao, Z. D. Cui, K. Kwok, and Y. Zhang. "Wake-induced vibration of a small cylinder in the wake of a large cylinder." Ocean Engineering, 113(1) (2016), pp. 75-89. DOI: $10.1016 / \mathrm{j}$.oceaneng.2015.12.032

29. M. Zhao and L. Cheng. "Numerical simulation of vortex-induced vibration of four circular cylinders in a square configuration." Journal of Fluids and Structures, 31(5) (2012), pp. 125-140. DOI: 10.1016/j.jfluidstructs.2012.04.002.

30. M. Zhao, K. Kaja, Y. Xiang, and L. Cheng. "Vortex-induced vibration of four cylinders in an in-line square configuration." Physics of Fluids, 28(2), 023602 (2012). DOI: $10.1063 / 1.4941774$.

31. K. Lam, W. Q. Gong, and R. M. C. So. "Numerical simulation of cross-flow around four cylinders in an in-line square configuration." Journal of Fluids and 
Structures, 24(1) (2008), pp. 34-57. DOI: 10.1016/j·jfluidstructs.2007.06. 003.

32. S. P. Singh and S. Mittal. "Vortex-induced oscillations at low Reynolds numbers: Hysteresis and vortex shedding modes." Journal of Fluids and Structures, 20(8) (2005), pp. 1085-1104. DOI: 10.1016/j·jfluidstructs.2005.05.011.

33. M. Zhao, K. Kaja, Y. Xiang, and L. Cheng. "Flow induced vibration of two rigidly coupled circular cylinders in tandem and side-by-side arrangements at a low Reynolds number of 150." Physics of Fluids, 25(12), 123601 (2013). DOI: $10.1063 / 1.4832956$

34. M. M. Zdravkovich. "The effects of interference between circular cylinders in cross flow." Journal of Fluids and Structures, 1(2) (1987), pp. 239-261. DOI: 10.1016/S0889-9746(87)90355-0.

35. Y. Zhou and M. W. Yiu. "Flow structure, momentum and heat transport in a two-tandem-cylinder wake." Journal of Fluid Mechanics, 548(2) (1987), pp. 1748. DOI: $10.1017 / \mathrm{S} 002211200500738 \mathrm{X}$. 